\title{
Criteria of asymptotic $\omega$-periodicity and their applications in a class of fractional differential equations
}

Rui Xie* and Chuanyi Zhang

"Correspondence:

xierui303030@126.com

Department of Mathematics, Harbin

Institute of Technology, Harbin,

150001, P.R. China

\begin{abstract}
In this paper we first characterize the (Stepanov) asymptotically $\omega$-periodic functions. Then we apply the criteria obtained to investigate the existence and uniqueness of asymptotically $\omega$-periodic mild solutions to semilinear fractional integro-differential equations with Stepanov asymptotically $\omega$-periodic coefficients.
\end{abstract}

MSC: $34 \mathrm{C} 25 ; 34 \mathrm{G} 20$

Keywords: asymptotically $\omega$-periodic functions; Stepanov asymptotically $\omega$-periodic functions; fractional differential equations

\section{Introduction}

The study of the existence of periodic solutions to differential equations is one of the most important topics in the qualitative theory, due both to its mathematical interest and its applications in many scientific fields, such as mathematical biology, control theory, physics, etc. However, some phenomena in the real world are not periodic, but approximately periodic or asymptotically periodic. As a result, in the past several decades many authors proposed and developed several extensions of the concept of periodicity, such as almost periodicity, almost automorphy, pseudo almost periodicity, pseudo almost automorphy, etc., and the same concept in the Stepanov sense (cf. [1-4] and references therein).

In this paper, $\mathbb{R}^{+}$is the interval $[0, \infty)$ and $X$ is a complex Banach space. Let $C_{b}\left(\mathbb{R}^{+}, X\right)$ denote the space consisting of bounded and continuous functions from $\mathbb{R}^{+}$into $X$, endowed with the uniform convergence norm $\|\cdot\|_{\infty}$. Set $C_{0}\left(\mathbb{R}^{+}, X\right)=\left\{f \in C_{b}\left(\mathbb{R}^{+}, X\right)\right.$ : $\left.\lim _{t \rightarrow \infty}\|f(t)\|=0\right\}$, and $P_{\omega}\left(\mathbb{R}^{+}, X\right)=\left\{f \in C_{b}\left(\mathbb{R}^{+}, X\right): f\right.$ is $\omega$ periodic $\}$. A function $f \in$ $C_{b}\left(\mathbb{R}^{+}, X\right)$ is said to be asymptotically $\omega$-periodic if it can be expressed as $f=g+h$, where $g \in P_{\omega}\left(\mathbb{R}^{+}, X\right)$ and $h \in C_{0}\left(\mathbb{R}^{+}, X\right)$. The collection of such functions will be denoted by $A P_{\omega}\left(\mathbb{R}^{+}, X\right)$. Let $f=g+h \in A P_{\omega}\left(\mathbb{R}^{+}, X\right)$. Since $g \in P_{\omega}\left(\mathbb{R}^{+}, X\right)$, one gets

$$
\lim _{t \rightarrow \infty}(f(t+\omega)-f(t))=\lim _{t \rightarrow \infty}(h(t+\omega)-h(t))=0 .
$$

The converse is not true. The authors in [5] provided two examples to show that there exist bounded continuous functions which satisfy (1.1), but are not asymptotically $\omega$ periodic. At the same time, (1.1) leads the authors in [6] to propose the notion: a function $f \in C_{b}\left(\mathbb{R}^{+}, X\right)$ is said to be $S$-asymptotically $\omega$-periodic if there exists $\omega>0$ such 
that $\lim _{t \rightarrow \infty}(f(t+\omega)-f(t))=0$. Obviously, $S$-asymptotic $\omega$-periodicity is more general than asymptotic $\omega$-periodicity. The authors in [6] discussed qualitative properties of such functions on Banach spaces and gave some conditions under which an $S$-asymptotically $\omega$-periodic function is asymptotically $\omega$-periodic. Nowadays, both asymptotically and $S$ asymptotically $\omega$-periodic functions have been applied to qualitative analysis for various kinds of problems. In [7], the authors studied the existence of $S$-asymptotically and asymptotically $\omega$-periodic solutions for some class of abstract neutral functional differential equations with infinite delay. In [8], the authors studied the existence and uniqueness of $S$-asymptotically and asymptotically $\omega$-periodic solutions to an abstract differential equation with linear part dominated by a Hille-Yosida operator with nondense domain. Pierri [9] discussed the existence of $S$-asymptotically and asymptotically $\omega$-periodic solutions to an abstract integral equation. In the recent paper [10], the authors studied the existence of asymptotically $\omega$-periodic solutions to a class of semilinear nonautonomous evolution equations involving nonlocal initial conditions. For further literature concerning this topic we refer the reader to $[1,11-21]$. The concept of $S$-asymptotic $\omega$-periodicity in the Stepanov sense was introduced in [22], Definition 1.3. For its applications, we refer the reader to $[18,22]$.

With respect to asymptotic periodicity, it is natural to ask: if there exists a necessary and sufficient condition for asymptotically $\omega$-periodic functions due to both its mathematical interest and its applications. Furthermore, if we consider asymptotically $\omega$-periodic functions in the Stepanov sense, it is also natural to ask: if there exists a necessary and sufficient condition for asymptotically $\omega$-periodic functions in the Stepanov sense. We remark that it is a natural idea to propose the concept of asymptotic $\omega$-periodicity in the Stepanov sense which extend the one of asymptotic $\omega$-periodicity to include locally integrable functions. Since the condition of continuity is not required, the concept of asymptotic $\omega$-periodicity in the Stepanov sense can describe the real world more realistically. However, as far as we know, there is only a little work which used the concept of asymptotic $\omega$-periodicity in the Stepanov sense ( $c f$. [22]).

We will characterize asymptotic $\omega$-periodicity and asymptotic $\omega$-periodicity in the Stepanov sense, respectively, in Sections 2 and 3. Finally in Section 4 we will apply the criteria to investigate the existence and uniqueness of asymptotically $\omega$-periodic mild solutions to semilinear fractional integro-differential equations with Stepanov asymptotically $\omega$-periodic coefficients.

\section{Criteria of asymptotically $\omega$-periodic functions}

Theorem 2.1 Let $f \in C_{b}\left(\mathbb{R}^{+}, X\right)$ and $\omega>0$. Then the following statements are equivalent:

(1) $f \in A P_{\omega}\left(\mathbb{R}^{+}, X\right)$;

(2) $g(t)=\lim _{n \rightarrow \infty} f(t+n \omega)$ uniform on $\mathbb{R}^{+}$;

(3) $g(t)=\lim _{n \rightarrow \infty} f(t+n \omega)$ uniformly on compact subsets of $\mathbb{R}^{+}$;

(4) $g(t)=\lim _{n \rightarrow \infty} f(t+n \omega)$ is well defined for each $t \in \mathbb{R}^{+}$and $g(t)=\lim _{n \rightarrow \infty} f(t+n \omega)$ uniformly on $[0, \omega]$.

Proof Clearly (2) implies (3) and (3) implies (4).

Suppose next that (1) holds and let $f=f_{1}+f_{2}$, where $f_{1} \in P_{\omega}\left(\mathbb{R}^{+}, X\right)$ and $f_{2} \in C_{0}\left(\mathbb{R}^{+}, X\right)$. Now for $n \in \mathbb{N}$, the periodicity of the function $f_{1}$ gives

$$
f(t+n \omega)=f_{1}(t+n \omega)+f_{2}(t+n \omega)=f_{1}(t)+f_{2}(t+n \omega) .
$$


Let $\varepsilon>0$. Since $f_{2} \in C_{0}\left(\mathbb{R}^{+}, X\right)$, there exists a positive integer $N_{1}$ such that $\left\|f_{2}(t+n \omega)\right\|<\varepsilon$ when $n \geq N_{1}$ for every $t \in \mathbb{R}^{+}$. Then, using (2.1), we conclude that $\left\|f(t+n \omega)-f_{1}(t)\right\|<\varepsilon$ when $n \geq N_{1}$ for every $t \in \mathbb{R}^{+}$, which shows that $f_{1}(t)=\lim _{n \rightarrow \infty} f(t+n \omega)$ uniformly on $\mathbb{R}^{+}$. Hence (1) implies (2).

Finally, suppose that (4) holds. It is clear $g$ is bounded on $\mathbb{R}^{+}$and $g(t+\omega)=g(t)$ for each $t \in \mathbb{R}^{+}$because $g(t+\omega)=\lim _{n \rightarrow \infty} f(t+\omega+(n-1) \omega)=\lim _{n \rightarrow \infty} f(t+n \omega)=g(t)$.

Thus, to show the continuity of the function $g$ on $\mathbb{R}^{+}$we only need to prove that $g$ is continuous on $[0, \omega]$.

Now, take any fixed $t_{0} \in[0, \omega]$ and let $t \in[0, \omega]$. For each $n \in \mathbb{N}$, we have

$$
\begin{aligned}
\left\|g(t)-g\left(t_{0}\right)\right\| \leq & \|g(t)-f(t+n \omega)\|+\left\|f(t+n \omega)-f\left(t_{0}+n \omega\right)\right\| \\
& +\left\|f\left(t_{0}+n \omega\right)-g\left(t_{0}\right)\right\| .
\end{aligned}
$$

Let $\varepsilon>0$. Then, by assumption in (4), we conclude that there exists a positive integer $N_{2}$ such that

$$
\|g(t)-f(t+n \omega)\|<\frac{\varepsilon}{3}
$$

for $t \in[0, \omega]$ and $n \geq N_{2}$.

On the other hand, since $f\left(t+N_{2} \omega\right) \in C_{b}\left(\mathbb{R}^{+}, X\right)$, then there exists $\delta>0$ such that

$$
\left\|f\left(t+N_{2} \omega\right)-f\left(t_{0}+N_{2} \omega\right)\right\|<\frac{\varepsilon}{3}
$$

for $\left|t-t_{0}\right|<\delta$.

Using (2.2), (2.3), and (2.4), we conclude that $\left\|g(t)-g\left(t_{0}\right)\right\|<\varepsilon$ when $\left|t-t_{0}\right|<\delta$, which shows that $g$ is continuous at $t_{0}$. Moreover, $g$ is continuous on $[0, \omega]$. Hence $g \in P_{\omega}\left(\mathbb{R}^{+}, X\right)$.

Next, we will show that $f-g \in C_{0}\left(\mathbb{R}^{+}, X\right)$. Suppose that $\varepsilon>0$ and there exists a positive integer $N_{3}$ such that $\|f(t+n \omega)-g(t)\|<\varepsilon$ when $n \geq N_{3}$ uniformly for $t \in[0, \omega]$ by assumption in (4) again.

Thus, for $n=N_{3}+k, k=0,1,2, \ldots$, we conclude that $\left\|f\left(t+\left(N_{3}+k\right) \omega\right)-g(t)\right\|<\varepsilon$ uniformly for $t \in[0, \omega]$. Moreover, if we denote $t^{\prime}=t+k \omega$, where $t^{\prime} \in[k \omega,(k+1) \omega]$ and $k=0,1,2, \ldots$, then we obtain

$$
\begin{aligned}
\left\|f\left(t^{\prime}+N_{3} \omega\right)-g\left(t^{\prime}+N_{3} \omega\right)\right\| & =\left\|f\left(t+\left(N_{3}+k\right) \omega\right)-g\left(t+\left(N_{3}+k\right) \omega\right)\right\| \\
& =\left\|f\left(t+\left(N_{3}+k\right) \omega\right)-g(t)\right\| \\
& <\varepsilon
\end{aligned}
$$

for $t^{\prime} \in[k \omega,(k+1) \omega], k=0,1,2, \ldots$ That is $\|f(t)-g(t)\|<\varepsilon\left(t \geq N_{3} \omega\right)$, which shows that $f-g \in C_{0}\left(\mathbb{R}^{+}, X\right)$. Hence $f \in A P_{\omega}\left(\mathbb{R}^{+}, X\right)$. This shows that (4) implies (1).

\section{Criteria of Stepanov asymptotically $\omega$-periodic functions}

Let $p \in[1, \infty)$ and $q$ denote the conjugate exponent of $p$.

The Bochner transform $f^{b}(t, s), t \in \mathbb{R}^{+}, s \in[0,1]$ of a function $f(t)$ on $\mathbb{R}^{+}$with values in $X$ is defined by $f^{b}(t, s)=f(t+s)$. 
Let $f: \mathbb{R}^{+} \rightarrow X$ be a measurable function. We say that $f$ is a Stepanov bounded function, with the exponent $p$, if $\sup _{t \in \mathbb{R}^{+}} \int_{t}^{t+1}\|f(s)\|^{p} d s<\infty$. The collection of such functions will be denoted by $B S^{p}\left(\mathbb{R}^{+}, X\right)$.

The space $B S^{p}\left(\mathbb{R}^{+}, X\right)$ endowed with the norm

$$
\|f\|_{S^{p}}=\left\|f^{b}\right\|_{L^{\infty}\left(\mathbb{R}^{+}, L^{p}(0,1 ; X)\right)}=\sup _{t \in \mathbb{R}^{+}}\left(\int_{t}^{t+1}\|f(s)\|^{p} d s\right)^{\frac{1}{p}}
$$

is a Banach space.

Define the subspaces of $B S^{p}\left(\mathbb{R}^{+}, X\right)$ by

$$
S^{p} P_{\omega}\left(\mathbb{R}^{+}, X\right)=\left\{f \in B S^{p}\left(\mathbb{R}^{+}, X\right): \int_{t}^{t+1}\|f(s+\omega)-f(s)\|^{p} d s=0, t \in \mathbb{R}^{+}\right\}
$$

and

$$
B S_{0}^{p}\left(\mathbb{R}^{+}, X\right)=\left\{f \in B S^{p}\left(\mathbb{R}^{+}, X\right): \lim _{t \rightarrow \infty} \int_{t}^{t+1}\|f(s)\|^{p} d s=0\right\}
$$

Now we give the definition of asymptotically $\omega$-periodic functions in the Stepanov sense.

Definition 3.1 A function $f \in B S^{p}\left(\mathbb{R}^{+}, X\right)$ is called asymptotically $\omega$-periodic in the Stepanov sense if it can be expressed as $f=g+h$, where $g \in S^{p} P_{\omega}\left(\mathbb{R}^{+}, X\right)$ and $h \in$ $B S_{0}^{p}\left(\mathbb{R}^{+}, X\right)$. The collection of such functions will be denoted by $S^{p} A P_{\omega}\left(\mathbb{R}^{+}, X\right)$.

There exists a function $a \in S^{p} A P_{\omega}\left(\mathbb{R}^{+}, X\right)$, but not in $A P_{\omega}\left(\mathbb{R}^{+}, X\right)$. For concision, we only consider the case $\omega=2 \pi$. Similarly one can exhibit examples for the general case $\omega>0$.

Example 3.2 Define the function $b: \mathbb{R}^{+} \rightarrow \mathbb{R}$ by

$$
b(t)= \begin{cases}\sin t, & t \in\left[2 k \pi, 2 k \pi+\frac{\pi}{2}\right], k=0,1, \ldots ; \\ 0, & \text { otherwise }\end{cases}
$$

and define the function $c: \mathbb{R}^{+} \rightarrow \mathbb{R}$ by $e^{-t}$. Let the function $a: \mathbb{R}^{+} \rightarrow \mathbb{R}$ defined by $a(t)=$ $b(t)+c(t)$ for each $t \in \mathbb{R}^{+}$.

We have $a \in S^{p} A P_{2 \pi}\left(\mathbb{R}^{+}, \mathbb{R}\right)$ because $b \in S^{p} P_{2 \pi}\left(\mathbb{R}^{+}, \mathbb{R}\right)$ and $c \in B S_{0}^{p}\left(\mathbb{R}^{+}, \mathbb{R}\right)$. However, it is not in $A P_{2 \pi}\left(\mathbb{R}^{+}, \mathbb{R}\right)$ because it is not continuous.

Next we give the criteria of asymptotically $\omega$-periodic functions in the Stepanov sense.

Theorem 3.3 Let $f \in L_{\mathrm{loc}}^{p}\left(\mathbb{R}^{+}, X\right)$ and $\omega>0$. Then the following statements are equivalent:

(1) $f \in S^{p} A P_{\omega}\left(\mathbb{R}^{+}, X\right)$;

(2) There exists a function $g \in S^{p} P_{\omega}\left(\mathbb{R}^{+}, X\right)$ such that $\int_{t}^{t+1}\|f(s+n \omega)-g(s)\|^{p} d s \rightarrow 0$ as $n \rightarrow \infty$ uniformly for $t \in \mathbb{R}^{+}$;

(3) There exists a function $g \in L_{\mathrm{loc}}^{p}\left(\mathbb{R}^{+}, X\right)$ such that $\int_{t}^{t+1}\|f(s+n \omega)-g(s)\|^{p} d s \rightarrow 0$ as $n \rightarrow \infty$ pointwise on $\mathbb{R}^{+}$. 
Proof Clearly (2) implies (3). Suppose next that (1) holds. Suppose that $f \in S^{p} A P_{\omega}\left(\mathbb{R}^{+}, X\right)$ and let $f=g+h$, where $g \in S^{p} P_{\omega}\left(\mathbb{R}^{+}, X\right)$ and $h \in B S_{0}^{p}\left(\mathbb{R}^{+}, X\right)$. Then we have

$$
\begin{aligned}
\left(\int_{t}^{t+1}\|f(s+n \omega)-g(s)\|^{p} d s\right)^{\frac{1}{p}} & \\
\leq & \left(\int_{t}^{t+1}\|f(s+n \omega)-g(s+n \omega)\|^{p} d s\right)^{\frac{1}{p}}+\left(\int_{t}^{t+1}\|g(s+n \omega)-g(s)\|^{p} d s\right)^{\frac{1}{p}} \\
\leq & \left(\int_{t}^{t+1}\|f(s+n \omega)-g(s+n \omega)\|^{p} d s\right)^{\frac{1}{p}} \\
& +\sum_{k=1}^{n}\left(\int_{t}^{t+1}\|g(s+k \omega)-g(s+(k-1) \omega)\|^{p} d s\right)^{\frac{1}{p}} \\
= & \left(\int_{t}^{t+1}\|h(s+n \omega)\|^{p} d s\right)^{\frac{1}{p}}+\sum_{k=1}^{n}\left(\int_{t+(k-1) \omega}^{t+(k-1) \omega+1}\|g(s+\omega)-g(s)\|^{p} d s\right)^{\frac{1}{p}} \\
= & \left(\int_{t+n \omega}^{t+n \omega+1}\|h(s)\|^{p} d s\right)^{\frac{1}{p}} \cdot
\end{aligned}
$$

Let $\varepsilon>0$. Since $h \in B S_{0}^{p}\left(\mathbb{R}^{+}, X\right)$, we conclude that there exists $N_{1} \in \mathbb{N}$ such that

$$
\left(\int_{t+n \omega}^{t+n \omega+1}\|h(s)\|^{p} d s\right)^{\frac{1}{p}}<\varepsilon
$$

when $n \geq N_{1}$ for all $t \in \mathbb{R}^{+}$. Therefore,

$$
\lim _{n \rightarrow \infty}\left(\int_{t}^{t+1}\|f(s+n \omega)-g(s)\|^{p} d s\right)^{\frac{1}{p}}=0
$$

uniformly for $t \in \mathbb{R}^{+}$. Hence (1) implies (2).

Now, suppose (3) holds. Then there exists $g \in L_{\text {loc }}^{p}\left(\mathbb{R}^{+}, X\right)$ such that

$$
\int_{t}^{t+1}\|f(s+n \omega)-g(s)\|^{p} d s \rightarrow 0
$$

as $n \rightarrow \infty$ pointwise on $\mathbb{R}^{+}$.

Let $\varepsilon>0$. For fixed $t \in \mathbb{R}^{+}$, one has

$$
\begin{aligned}
& \left(\int_{t}^{t+1}\|g(s+\omega)-g(s)\|^{p} d s\right)^{\frac{1}{p}} \\
& \quad \leq\left(\int_{t}^{t+1}\|f(s+n \omega)-g(s)\|^{p} d s\right)^{\frac{1}{p}}+\left(\int_{t}^{t+1}\|f((s+\omega)+(n-1) \omega)-g(s+\omega)\|^{p} d s\right)^{\frac{1}{p}} \\
& \quad=\left(\int_{t}^{t+1}\|f(s+n \omega)-g(s)\|^{p} d s\right)^{\frac{1}{p}}+\left(\int_{t+\omega}^{t+\omega+1}\|f(s+(n-1) \omega)-g(s)\|^{p} d s\right)^{\frac{1}{p}} .
\end{aligned}
$$

By (3.1) there exists $N_{2} \in \mathbb{N}$ such that

$$
\left(\int_{t}^{t+1}\|f(s+n \omega)-g(s)\|^{p} d s\right)^{\frac{1}{p}}<\frac{\varepsilon}{2}
$$


Xis and Chang Advances in Difference Equations ( 2015) 2015:68

Page 6 of 20

and

$$
\left(\int_{t+\omega}^{t+\omega+1}\|f(s+(n-1) \omega)-g(s)\|^{p} d s\right)^{\frac{1}{p}}<\frac{\varepsilon}{2}
$$

when $n \geq N_{2}$. Equations (3.2) and (3.3) imply

$$
\left(\int_{t}^{t+1}\|g(s+\omega)-g(s)\|^{p} d s\right)^{\frac{1}{p}}<\varepsilon
$$

which shows

$$
\left(\int_{t}^{t+1}\|g(s+\omega)-g(s)\|^{p} d s\right)^{\frac{1}{p}}=0
$$

for every $t \in \mathbb{R}^{+}$. It follows from (3.4) that

$$
\left(\int_{t+m \omega}^{t+m \omega+1}\|g(s)\|^{p} d s\right)^{\frac{1}{p}}=\left(\int_{t}^{t+1}\|g(s)\|^{p} d s\right)^{\frac{1}{p}}
$$

for any $m \in \mathbb{N}$. For,

$$
\begin{aligned}
\left(\int_{t+\omega}^{t+\omega+1}\|g(s)\|^{p} d s\right)^{\frac{1}{p}} & =\left(\int_{t}^{t+1}\|g(s+\omega)\|^{p} d s\right)^{\frac{1}{p}} \\
& \leq\left(\int_{t}^{t+1}\|g(s)\|^{p} d s\right)^{\frac{1}{p}}+\left(\int_{t}^{t+1}\|g(s+\omega)-g(s)\|^{p} d s\right)^{\frac{1}{p}} \\
& =\left(\int_{t}^{t+1}\|g(s)\|^{p} d s\right)^{\frac{1}{p}}
\end{aligned}
$$

In the same way, we have

$$
\left(\int_{t}^{t+1}\|g(s)\|^{p} d s\right)^{\frac{1}{p}} \leq\left(\int_{t+\omega}^{t+\omega+1}\|g(s)\|^{p} d s\right)^{\frac{1}{p}} .
$$

So

$$
\left(\int_{t+\omega}^{t+\omega+1}\|g(s)\|^{p} d s\right)^{\frac{1}{p}}=\left(\int_{t}^{t+1}\|g(s)\|^{p} d s\right)^{\frac{1}{p}}
$$

and (3.5) holds.

In the following of the proof, $n_{0} \in \mathbb{N}$ is a fixed number such that $n_{0} \geq \omega$. For every $t \in \mathbb{R}^{+}$, we can write $t=N(t) \omega+s$, where $N(t) \in \mathbb{N}$ and $s \in(0, \omega)$. By (3.5), we have

$$
\begin{aligned}
\int_{t}^{t+1}\|g(s)\|^{p} d s & =\int_{N(t) \omega+s}^{N(t) \omega+s+1}\|g(s)\|^{p} d s \\
& =\int_{s}^{s+1}\|g(s)\|^{p} d s \\
& \leq \int_{0}^{n_{0}+1}\|g(s)\|^{p} d s .
\end{aligned}
$$


Since $g \in L_{\text {loc }}^{p}\left(\mathbb{R}^{+}, X\right)$, there exists $M>0$ such that $\int_{0}^{n_{0}+1}\|g(s)\|^{p} d s \leq M$. Then we obtain

$$
\sup _{t \in \mathbb{R}^{+}} \int_{t}^{t+1}\|g(s)\|^{p} d s \leq M
$$

which shows $g \in B S^{p}\left(\mathbb{R}^{+}, X\right)$. Combining with (3.4), we know that $g \in S^{p} P_{\omega}\left(\mathbb{R}^{+}, X\right)$.

Let $h(s)=f(s)-g(s), s \in \mathbb{R}^{+}$. For fixed $t \in \mathbb{R}^{+}$and any $\varepsilon>0$, by (3.1) there exists $N_{3}(t) \in \mathbb{N}$ such that

$$
\left(\int_{t}^{t+1}\|f(s+n \omega)-g(s)\|^{p} d s\right)^{\frac{1}{p}}<\varepsilon
$$

when $n \geq N_{3}(t)$. Then we have

$$
\begin{aligned}
\left(\int_{t+n \omega}^{t+1+n \omega}\|h(s)\|^{p} d s\right)^{\frac{1}{p}}= & \left(\int_{t}^{t+1}\|h(s+n \omega)\|^{p} d s\right)^{\frac{1}{p}} \\
= & \left(\int_{t}^{t+1}\|f(s+n \omega)-g(s+n \omega)\|^{p} d s\right)^{\frac{1}{p}} \\
\leq & \left(\int_{t}^{t+1}\|f(s+n \omega)-g(s)\|^{p} d s\right)^{\frac{1}{p}} \\
& +\left(\int_{t}^{t+1}\|g(s+n \omega)-g(s)\|^{p} d s\right)^{\frac{1}{p}} .
\end{aligned}
$$

Note that

$$
\left(\int_{t}^{t+1}\|g(s+n \omega)-g(s)\|^{p} d s\right)^{\frac{1}{p}}=0 .
$$

From (3.6) we have

$$
\left(\int_{t+n \omega}^{t+1+n \omega}\|h(s)\|^{p} d s\right)^{\frac{1}{p}}<\varepsilon
$$

when $n \geq N_{3}(t)$.

Next, we will prove that there exists $N_{4} \in \mathbb{N}$ such that for any $\tau \in(0, \omega)$

$$
\left(\int_{\tau+n \omega}^{\tau+1+n \omega}\|h(s)\|^{p} d s\right)^{\frac{1}{p}}<\varepsilon
$$

when $n \geq N_{4}$.

For $\tau \in(0, \omega)$, we have

$$
\int_{\tau+n \omega}^{\tau+1+n \omega}\|h(s)\|^{p} d s \leq \sum_{k=0}^{n_{0}} \int_{k+n \omega}^{k+1+n \omega}\|h(s)\|^{p} d s .
$$

Let $N_{4}=\max \left\{N_{3}(0), N_{3}(1), \ldots, N_{3}\left(n_{0}\right)\right\}$. Replace $\varepsilon$ in $(3.7)$ by $\varepsilon /\left(n_{0}+1\right)^{\frac{1}{p}}$, then

$$
\left(\int_{k+n \omega}^{k+1+n \omega}\|h(s)\|^{p} d s\right)^{\frac{1}{p}}<\frac{\varepsilon}{\left(n_{0}+1\right)^{\frac{1}{p}}}
$$


when $n \geq N_{3}(k)$ for $k=0,1,2, \ldots, n_{0}$. Equations (3.9) and (3.10) imply (3.8). For every $t \in$ $\mathbb{R}^{+}$, again $t=N(t) \omega+\tau$, where $N(t) \in \mathbb{N}$ and $\tau \in(0, \omega)$. Thus

$$
\left(\int_{t+n \omega}^{t+1+n \omega}\|h(s)\|^{p} d s\right)^{\frac{1}{p}}=\left(\int_{N(t) \omega+\tau+n \omega}^{N(t) \omega+\tau+1+n \omega}\|h(s)\|^{p} d s\right)^{\frac{1}{p}}=\left(\int_{\tau+(N(t)+n) \omega}^{\tau+1+(N(t)+n) \omega}\|h(s)\|^{p} d s\right)^{\frac{1}{p}} .
$$

Combining with (3.8), one gets

$$
\left(\int_{t+n \omega}^{t+1+n \omega}\|h(s)\|^{p} d s\right)^{\frac{1}{p}}<\varepsilon
$$

when $n \geq N_{4}$ for all $t \in \mathbb{R}^{+}$. That is $\left(\int_{t}^{t+1}\|h(s)\|^{p} d s\right)^{\frac{1}{p}}<\varepsilon\left(t \geq N_{4} \omega\right)$, which shows $h \in$ $B S_{0}^{p}\left(\mathbb{R}^{+}, X\right)$. Therefore $f=g+h$, where $g \in S^{p} P_{\omega}\left(\mathbb{R}^{+}, X\right)$ and $h \in B S_{0}^{p}\left(\mathbb{R}^{+}, X\right)$, that is, $f \in$ $S^{p} A P_{\omega}\left(\mathbb{R}^{+}, X\right)$. This shows that (3) implies (1).

\section{Asymptotically $\omega$-periodic solutions of semilinear fractional integro-differential equations}

In this section, we will investigate the following semilinear fractional integro-differential equations:

$$
\left\{\begin{array}{l}
v^{\prime}(t)=\int_{0}^{t} \frac{(t-s)^{\alpha-2}}{\Gamma(\alpha-1)} A v(s) d s+f(t, v(t)), \quad t \in \mathbb{R}^{+} \\
v(0)=v_{0} \in X
\end{array}\right.
$$

where $1<\alpha<2, A: D(A) \subset X \rightarrow X$ is a linear densely defined operator of sectorial type on a complex Banach space $X$ and $f: \mathbb{R}^{+} \times X \rightarrow X$ is an appropriate function. In particular, the convolution integral in (4.1) is known as the Riemann-Liouville integral. For more details we refer the reader to [23].

Recently, fractional differential equations have attracted much attention (see [13-18, 23-27] and references therein) due to their applications in various fields of science and engineering. In particular, the authors in [13] studied the existence and uniqueness of $S$ asymptotically $\omega$-periodic mild solution of the problem (4.1) when the nonlinear term $f$ is $S$-asymptotically $\omega$-periodic. Moreover, if the continuous nonlinear term $f$ satisfies some stronger conditions (see [13], Theorem 3.7), the authors proved that the problem (4.1) has a unique asymptotically $\omega$-periodic mild solution. Later, the author in [18] studied the existence and uniqueness of $S$-asymptotically $\omega$-periodic mild solution of the problem (4.1) when the nonlinear perturbation is $S$-asymptotically $\omega$-periodic in the Stepanov sense.

However, to the best of our knowledge, there is no work reported in literature on conditions under which the problem (4.1) has a unique asymptotically $\omega$-periodic mild solution when the nonlinear perturbation is $S$-asymptotically or asymptotically $\omega$-periodic in the Stepanov sense. Now we will apply the results obtained in Sections 2 and 3 to show that the problem (4.1) has a unique asymptotically $\omega$-periodic mild solution as long as the nonlinear perturbation is asymptotically $\omega$-periodic functions in the Stepanov sense and satisfies a Lipschitz condition.

First let us recall some definitions about sectorial linear operators.

Definition 4.1 [28] A closed and dense defined linear operator $A$ is called sectorial of type $\mu$ if there exist $0<\theta<\frac{\pi}{2}, M>0$, and $\mu \in \mathbb{R}$ such that its resolvent exists outside the 
sector

$$
\mu+S_{\theta}=\{\mu+\lambda: \lambda \in \mathbb{C},|\arg (-\lambda)|<\theta\}
$$

and $\left\|(\lambda I-A)^{-1}\right\| \leq \frac{M}{|\lambda-\mu|}, \lambda \notin \mu+S_{\theta}$.

Definition 4.2 [24] Let $A$ be a closed and linear operator with domain $D(A)$ defined on a Banach space $X$. We call $A$ the generator of a solution operator if there exist $\mu \in \mathbb{R}$ and a strong continuous function $S_{\alpha}: \mathbb{R}^{+} \rightarrow L(X)$ such that $\left\{\lambda^{\alpha}: \operatorname{Re} \lambda>\mu\right\} \subset \rho(A)$ and

$$
\lambda^{\alpha-1}\left(\lambda^{\alpha}-A\right)^{-1} x=\int_{0}^{\infty} e^{-\lambda t} S_{\alpha}(t) x d t, \quad \operatorname{Re} \lambda>\mu, x \in X .
$$

In this case, $S_{\alpha}(t)$ is called the solution operator generated by $A$. In particular, $S_{\alpha}(0)=I$ [29], Proposition 2.6. We remark that the power function $\lambda^{\alpha}$ is uniquely defined as $\lambda^{\alpha}=$ $|\lambda|^{\alpha} e^{i \alpha \arg (\lambda)}$, with $-\pi<\arg (\lambda)<\pi$.

If $A$ is sectorial of type $\mu$ with $0<\theta<\pi\left(1-\frac{\alpha}{2}\right)$, then $A$ is the generator of a solution operator given by

$$
S_{\alpha}(t):=\frac{1}{2 \pi i} \int_{\gamma} e^{\lambda t} \lambda^{\alpha-1}\left(\lambda^{\alpha}-A\right)^{-1} d \lambda,
$$

where $\gamma$ is a suitable path lying outside the sector $\mu+S_{\theta}(c f .[23,24])$. Cuesta in [23] proved that $A$ is sectorial operator of type $\mu<0$ for some $0<\theta<\pi\left(1-\frac{\alpha}{2}\right)(1<\alpha<2), M>0$, then there exists a constant $C>0$ such that

$$
\left\|S_{\alpha}(t)\right\| \leq \frac{C M}{1+|\mu| t^{\alpha}}, \quad t \in \mathbb{R}^{+} .
$$

Note that

$$
\int_{0}^{\infty} \frac{1}{1+|\mu| t^{\alpha}} d t=\frac{|\mu|^{-\frac{1}{\alpha}} \pi}{\alpha \sin \left(\frac{\pi}{\alpha}\right)}
$$

for $1<\alpha<2$ and therefore $S_{\alpha}(t)$ is integrable on $(0, \infty)$.

Next we give some necessary basic definitions of fractional calculus.

Definition 4.3 [30] The fractional order integral of order $\alpha>0$ with the low limit $t_{0}>0$ for a function $f$ is defined as

$$
I^{\alpha} f(t)=\frac{1}{\Gamma(\alpha)} \int_{t_{0}}^{t}(t-s)^{\alpha-1} f(s) d s,
$$

where $t>t_{0}, \alpha>0$, provided the right-hand side is pointwise defined on $\left[t_{0}, \infty\right)$, where $\Gamma$ is the gamma function.

Definition 4.4 [30] Riemann-Liouville derivative of order $\alpha>0$ with the low limit $t_{0}>0$ for a function $f:\left[t_{0}, \infty\right) \rightarrow \mathbb{R}$ can be written as

$$
D_{t}^{\alpha} f(t)=\frac{1}{\Gamma(n-\alpha)} \frac{d^{n}}{d t^{n}} \int_{t_{0}}^{t}(t-s)^{n-\alpha-1} f(s) d s,
$$

where $t>t_{0}$ and $n-1<\alpha<n$. 
For more details we refer the reader to [13, 23, 30]. We remark that a fractional calculus approach to the description of stress and strain localization in fractal media was introduced in [31]. If $A$ generates a solution operator, the variation of parameters formula allows us to write the solution of the problem (4.1) as $v(t)=S_{\alpha}(t) v_{0}+\int_{0}^{t} S_{\alpha}(t-s) f(s, v(s)) d s$, $t \in \mathbb{R}^{+}$. This motivates the authors in [13] to propose the following definition.

Definition 4.5 ([13], Definition 3.1) Suppose $A$ generates an integrable solution operator $S_{\alpha}(t)$. A function $v \in C_{b}\left(\mathbb{R}^{+}, X\right)$ is said to be a mild solution of the problem (4.1) if

$$
v(t)=S_{\alpha}(t) v_{0}+\int_{0}^{t} S_{\alpha}(t-s) f(s, v(s)) d s, \quad t \in \mathbb{R}^{+} .
$$

We need the following lemma.

Lemma 4.6 Suppose $f \in S^{p} A P_{\omega}\left(\mathbb{R}^{+}, X\right), f=g+h$ where $g \in S^{p} P_{\omega}\left(\mathbb{R}^{+}, X\right)$ and $h \in B S_{0}^{p}\left(\mathbb{R}^{+}\right.$, $X)$. Let $\omega=n_{0}+\theta$, where $n_{0} \in \mathbb{N}$ and $\theta \in(0,1)$. Then the following statements are true.

(1) $\int_{t}^{t+\omega}\|f(s)\| d s \leq\left(n_{0}+1\right)\|f\|_{s p}$ for each $t \in \mathbb{R}^{+}$;

(2) $\int_{t}^{t+\omega}\|g(s+m \omega)-g(s)\| d s=0$ for each $t \in \mathbb{R}^{+}$and any $m \in \mathbb{N}$;

(3) $\lim _{n \rightarrow \infty} \int_{t}^{t+\omega}\|h(s+n)\| d s=0$ uniformly for $t \in \mathbb{R}^{+}$.

Proof First we can estimate

$$
\begin{aligned}
\int_{t}^{t+\omega}\|f(s)\| d s & \leq \int_{t}^{t+n_{0}+1}\|f(s)\| d s \\
& =\sum_{k=0}^{n_{0}} \int_{t+k}^{t+k+1}\|f(s)\| d s \\
& \leq \sum_{k=0}^{n_{0}}\left(\int_{t+k}^{t+k+1}\|f(s)\|^{p} d s\right)^{\frac{1}{p}} \\
& \leq\left(n_{0}+1\right)\|f\|_{S^{p}} .
\end{aligned}
$$

This shows (1). Similarly we have

$$
\int_{t}^{t+\omega}\|g(s+\omega)-g(s)\| d s \leq \sum_{k=0}^{n_{0}}\left(\int_{t+k}^{t+k+1}\|g(s+\omega)-g(s)\|^{p}\right)^{\frac{1}{p}},
$$

which shows (2). We also get

$$
\int_{t}^{t+\omega}\|h(s+n)\| d s=\int_{t+n}^{t+n+\omega}\|h(s)\| d s \leq \sum_{k=0}^{n_{0}}\left(\int_{t+n+k}^{t+n+k+1}\|h(s)\|^{p}\right)^{\frac{1}{p}}
$$

which implies (3).

The next result is the key to proving our main theorems.

Lemma 4.7 Let $\{S(t)\}_{t \geq 0} \subset B(X)$ be a strongly continuous family of bounded and linear operators such that $\|S(t)\| \leq \phi(t), t \in \mathbb{R}^{+}$, where $\phi \in L^{1}\left(\mathbb{R}^{+}\right)$is nonincreasing. Let $f \in S^{p} A P_{\omega}\left(\mathbb{R}^{+}, X\right)$, then $u(t)=\int_{0}^{t} S(t-s) f(s) d s \in A P_{\omega}\left(\mathbb{R}^{+}, X\right)$. 
Proof First we have $u \in C_{b}\left(\mathbb{R}^{+}, X\right)[18]$, Lemma 2.5. Note that

$$
\begin{aligned}
u(t+n \omega) & =\int_{0}^{t+n \omega} S(t+n \omega-s) f(s) d s \\
& =\int_{-n \omega}^{t} S(t-s) f(s+n \omega) d s \\
& =\int_{-n \omega}^{0} S(t-s) f(s+n \omega) d s+\int_{0}^{t} S(t-s) f(s+n \omega) d s \\
& =\int_{0}^{n \omega} S(t+s) f(n \omega-s) d s+\int_{0}^{t} S(t-s) f(s+n \omega) d s \\
& =I_{1}(t, n)+I_{2}(t, n) .
\end{aligned}
$$

We consider the terms $I_{i}(t, n)(i=1,2)$ separately. First we will prove that $I_{1}(t, n)$ is a Cauchy sequence in $X$ for each $t \in \mathbb{R}^{+}$.

For any $p \in \mathbb{N}, n \in \mathbb{N}$, one has

$$
\begin{aligned}
I_{1}(t, n+p)-I_{1}(t, n)= & \int_{0}^{(n+p) \omega} S(t+s) f((n+p) \omega-s) d s-\int_{0}^{n \omega} S(t+s) f(n \omega-s) d s \\
= & \int_{n \omega}^{(n+p) \omega} S(t+s) f((n+p) \omega-s) d s \\
& +\int_{0}^{n \omega} S(t+s)[f((n+p) \omega-s)-f(n \omega-s)] d s \\
= & I_{3}(t, n, p)+I_{4}(t, n, p) .
\end{aligned}
$$

We see that

$$
\begin{aligned}
\left\|I_{3}(t, n, p)\right\| & \leq \int_{0}^{p \omega}\|S(t+s+n \omega)\|\|f(p \omega-s)\| d s \\
& \leq \int_{0}^{p \omega} \phi(t+s+n \omega)\|f(p \omega-s)\| d s \\
& =\sum_{k=0}^{p-1} \int_{k \omega}^{(k+1) \omega} \phi(t+s+n \omega)\|f(p \omega-s)\| d s \\
& \leq \sum_{k=0}^{p-1} \phi(t+k \omega+n \omega) \int_{k \omega}^{(k+1) \omega}\|f(p \omega-s)\| d s .
\end{aligned}
$$

Then by Lemma 4.6(1), one has

$$
\begin{aligned}
\left\|I_{3}(t, n, p)\right\| & \leq\left(n_{0}+1\right)\|f\|_{S^{p}} \sum_{k=0}^{p-1} \phi(t+k \omega+n \omega) \\
& \leq \frac{\left(n_{0}+1\right)\|f\|_{S^{p}}}{\omega}\left(\int_{t+(n-1) \omega}^{t+n \omega} \phi(s) d s+\cdots+\int_{t+(p-2) \omega+n \omega}^{t+(p-1) \omega+n \omega} \phi(s) d s\right) \\
& \leq \frac{\left(n_{0}+1\right)\|f\|_{S^{p}}}{\omega} \int_{t+(n-1) \omega}^{\infty} \phi(s) d s .
\end{aligned}
$$


Let $\varepsilon>0$. Since $\phi \in L_{1}\left(\mathbb{R}^{+}\right)$, there exists $N_{1} \in \mathbb{N}$ such that $\int_{\left(N_{1}-1\right) \omega}^{\infty} \phi(s) d s<\frac{\omega \varepsilon}{\left(n_{0}+1\right)\|f\|_{S}}$. Then one gets $\left\|I_{3}(t, n, p)\right\|<\varepsilon$ when $n \geq N_{1}$ uniformly for $t \in \mathbb{R}^{+}$.

For $n \geq N_{1}$, we see that

$$
\begin{aligned}
I_{4}(t, n, p)= & \int_{0}^{N_{1} \omega} S(t+s)[f((n+p) \omega-s)-f(n \omega-s)] d s \\
& +\int_{N_{1} \omega}^{n \omega} S(t+s)[f((n+p) \omega-s)-f(n \omega-s)] d s \\
= & I_{5}(t, n, p)+I_{6}(t, n, p) .
\end{aligned}
$$

Now we estimate the term $I_{5}(t, n, p)$ :

$$
\begin{aligned}
\left\|I_{5}(t, n, p)\right\| & \leq \int_{0}^{N_{1} \omega}\|S(t+s)\|\|f((n+p) \omega-s)-f(n \omega-s)\| d s \\
& \leq \int_{0}^{N_{1} \omega} \phi(t+s)\|f((n+p) \omega-s)-f(n \omega-s)\| d s \\
& \leq \phi(0) \sum_{k=0}^{N_{1}-1} \int_{k \omega}^{(k+1) \omega}\|f((n+p) \omega-s)-f(n \omega-s)\| d s .
\end{aligned}
$$

Since $f \in S^{p} A P_{\omega}\left(\mathbb{R}^{+}, X\right)$, it can be expressed as $f=g+h$, where $g \in S^{p} P_{\omega}\left(\mathbb{R}^{+}, X\right)$ and $h \in$ $B S_{0}^{p}\left(\mathbb{R}^{+}, X\right)$. Then one has

$$
\begin{aligned}
\left\|I_{5}(t, n, p)\right\| \leq & \phi(0) \sum_{k=0}^{N_{1}-1}\left[\int_{k \omega}^{(k+1) \omega}\|g((n+p) \omega-s)-g(n \omega-s)\| d s\right. \\
& \left.+\int_{k \omega}^{(k+1) \omega}\|h((n+p) \omega-s)\| d s+\int_{k \omega}^{(k+1) \omega}\|h(n \omega-s)\| d s\right] \\
= & \phi(0) \sum_{k=0}^{N_{1}-1}\left[\int_{n \omega-(k+1) \omega}^{n \omega-k \omega}\|g(s+p \omega)-g(s)\| d s\right. \\
& \left.+\int_{0}^{\omega}\|h(s+((n+p)-(k+1) \omega))\| d s+\int_{0}^{\omega}\|h(s+(n-(k+1) \omega))\| d s\right] .
\end{aligned}
$$

By Lemma 4.6(2), we get

$$
\begin{aligned}
\left\|I_{5}(t, n, p)\right\| \leq & \phi(0) \sum_{k=0}^{N_{1}-1}\left[\int_{0}^{\omega}\|h(s+(n-(k+1) \omega))\| d s\right. \\
& \left.+\int_{0}^{\omega}\|h(s+((n+p)-(k+1) \omega))\| d s\right] .
\end{aligned}
$$

By Lemma 4.6(3), we can choose $N_{2} \in \mathbb{N}$ such that $N_{2} \geq N_{1}$ and

$$
\phi(0) \sum_{k=0}^{N_{1}-1}\left[\int_{0}^{\omega}\|h(s+(n-(k+1) \omega))\| d s+\int_{0}^{\omega}\|h(s+((n+p)-(k+1) \omega))\| d s\right]<\varepsilon
$$

when $n \geq N_{2}$. That is $\left\|I_{5}(t, n, p)\right\|<\varepsilon\left(n \geq N_{2}\right)$ uniformly for $t \in \mathbb{R}^{+}$. 
Next we estimate the term $I_{6}(t, n, p)$ :

$$
\begin{aligned}
\left\|I_{6}(t, n, p)\right\| & \leq \int_{N_{1} \omega}^{n \omega}\|S(t+s)\|\|f((n+p) \omega-s)-f(n \omega-s)\| d s \\
& \leq \int_{N_{1} \omega}^{n \omega} \phi(t+s)\|f((n+p) \omega-s)-f(n \omega-s)\| d s \\
& =\sum_{k=N_{1}}^{n-1} \int_{k \omega}^{(k+1) \omega} \phi(t+s)\|f((n+p) \omega-s)-f(n \omega-s)\| d s \\
& \leq \sum_{k=N_{1}}^{n-1} \phi(t+k \omega) \int_{k \omega}^{(k+1) \omega}\|f((n+p) \omega-s)-f(n \omega-s)\| d s \\
& \leq \sum_{k=N_{1}}^{n-1} \phi(t+k \omega)\left[\int_{k \omega}^{(k+1) \omega}\|f((n+p) \omega-s)\| d s+\int_{k \omega}^{(k+1) \omega}\|f(n \omega-s)\| d s\right] .
\end{aligned}
$$

By Lemma 4.6(1), we obtain

$$
\begin{aligned}
\left\|I_{6}(t, n, p)\right\| & \leq 2\left(n_{0}+1\right)\|f\|_{S^{p}} \sum_{k=N_{1}}^{n-1} \phi(t+k \omega) \\
& \leq \frac{2\left(n_{0}+1\right)\|f\|_{S^{p}}}{\omega}\left(\int_{t+\left(N_{1}-1\right) \omega}^{t+N_{1} \omega} \phi(s) d s+\cdots+\int_{t+(n-2) \omega}^{t+(n-1) \omega} \phi(s) d s\right) \\
& \leq \frac{2\left(n_{0}+1\right)\|f\|_{S^{p}}}{\omega} \int_{t+\left(N_{1}-1\right) \omega}^{\infty} \phi(s) d s \\
& <2 \varepsilon
\end{aligned}
$$

uniformly for $t \in \mathbb{R}^{+}$.

Thus, $\left\|I_{1}(t, n+p)-I_{1}(t, n)\right\| \leq\left\|I_{3}(t, n, p)\right\|+\left\|I_{5}(t, n, p)\right\|+\left\|I_{6}(t, n, p)\right\|<4 \varepsilon$ when $n \geq N_{2}$. This shows that $I_{1}(t, n)$ is a Cauchy sequence and we denote $\lim _{n \rightarrow \infty} I_{1}(t, n)$ by $F(t)$ for each $t \in \mathbb{R}^{+}$. Besides, from the proof we also know that $\lim _{n \rightarrow \infty} I_{1}(t, n)=F(t)$ uniformly for $t \in \mathbb{R}^{+}$.

Finally, we consider the term $I_{2}(t, n)$. Since $f \in S^{p} A P_{\omega}\left(\mathbb{R}^{+}, X\right), f=g+h$, where $g \in$ $S^{p} P_{\omega}\left(\mathbb{R}^{+}, X\right)$ and $h \in B S_{0}^{p}\left(\mathbb{R}^{+}, X\right)$, by Theorem 3.3(2), we have

$$
\lim _{n \rightarrow \infty}\left(\int_{t}^{t+1}\|f(s+n \omega)-g(s)\|^{p} d s\right)^{\frac{1}{p}}=0
$$

uniformly for $t \in \mathbb{R}^{+}$. We also have $I_{2}(t, n), \int_{0}^{t} S(t-s) g(s) d s \in C_{b}\left(\mathbb{R}^{+}, X\right)$, which is like the case of $u$.

For $m \leq t<m+1, m \in \mathbb{N}$, one has

$$
\begin{gathered}
\left\|I_{2}(t, n)-\int_{0}^{t} S(t-s) g(s) d s\right\| \\
\quad \leq \int_{0}^{t}\|S(t-s)\|\|f(s+n \omega)-g(s)\| d s \\
\quad \leq \int_{0}^{t} \phi(t-s)\|f(s+n \omega)-g(s)\| d s
\end{gathered}
$$




$$
\begin{aligned}
& =\int_{0}^{m} \phi(t-s)\|f(s+n \omega)-g(s)\| d s+\int_{m}^{t} \phi(t-s)\|f(s+n \omega)-g(s)\| d s \\
& \leq \sum_{k=0}^{m-1} \int_{k}^{k+1} \phi(t-s)\|f(s+n \omega)-g(s)\| d s+\phi(0) \int_{m}^{t}\|f(s+n \omega)-g(s)\| d s \\
& \leq \sum_{k=0}^{m-1} \phi(t-(k+1)) \int_{k}^{k+1}\|f(s+n \omega)-g(s)\| d s+\phi(0) \int_{m}^{m+1}\|f(s+n \omega)-g(s)\| d s .
\end{aligned}
$$

By the Hölder inequality, we obtain

$$
\begin{aligned}
\left\|I_{2}(t, n)-\int_{0}^{t} S(t-s) g(s) d s\right\| \\
\leq \sum_{k=0}^{m-1} \phi(t-(k+1))\left(\int_{k}^{k+1}\|f(s+n \omega)-g(s)\|^{p} d s\right)^{\frac{1}{p}} \\
\quad+\phi(0)\left(\int_{m}^{m+1}\|f(s+n \omega)-g(s)\|^{p} d s\right)^{\frac{1}{p}} \\
\leq(\phi(t-1)+\cdots+\phi(t-m)+\phi(0)) \sup _{t \in \mathbb{R}^{+}}\left(\int_{t}^{t+1}\|f(s+n \omega)-g(s)\|^{p} d s\right)^{\frac{1}{p}} \\
\leq\left(\int_{t-2}^{t-1} \phi(s) d s+\cdots+\int_{t-m}^{t-(m-1)} \phi(s) d s+2 \phi(0)\right) \\
\quad \times \sup _{t \in \mathbb{R}^{+}}\left(\int_{t}^{t+1}\|f(s+n \omega)-g(s)\|^{p} d s\right)^{\frac{1}{p}} \\
\leq\left(\|\phi\|_{L^{1}}+2 \phi(0)\right) \sup _{t \in \mathbb{R}^{+}}\left(\int_{t}^{t+1}\|f(s+n \omega)-g(s)\|^{p} d s\right)^{\frac{1}{p}} .
\end{aligned}
$$

Then from (4.4), it follows that

$$
\lim _{n \rightarrow \infty} I_{2}(t, n)=\int_{0}^{t} S(t-s) g(s) d s
$$

uniformly for $t \in \mathbb{R}^{+}$.

Therefore,

$$
\lim _{n \rightarrow \infty} u(t+n \omega)=\lim _{n \rightarrow \infty} I_{1}(t, n)+\lim _{n \rightarrow \infty} I_{2}(t, n)=F(t)+\int_{0}^{t} S(t-s) g(s) d s
$$

uniformly for $t \in \mathbb{R}^{+}$. By Theorem 2.1, we have $u \in A P_{\omega}\left(\mathbb{R}^{+}, X\right)$.

To establish our results we introduce the following definition.

Definition 4.8 A function $f: \mathbb{R}^{+} \times X \rightarrow X$ with $f(t, x) \in L_{\mathrm{loc}}^{p}\left(\mathbb{R}^{+}, X\right)$ for each $x \in X$ is said to be asymptotically $\omega$-periodic in the Stepanov sense uniformly on bounded sets of $X$ if there exists a function $g: \mathbb{R}^{+} \times X \rightarrow X$ with $g(t, x) \in S^{p} P_{\omega}\left(\mathbb{R}^{+}, X\right)$ for each $x \in X$ such that for every bounded set $K \subset X$ we have

$$
\left(\int_{t}^{t+1}\|f(s+n \omega, x)-g(s, x)\|^{p} d s\right)^{\frac{1}{p}} \rightarrow 0
$$


as $n \rightarrow \infty$ pointwise on $\mathbb{R}^{+}$uniformly for $x \in K$. The collection of such functions will be denoted by $S^{p} A P_{\omega}\left(\mathbb{R}^{+} \times X, X\right)$.

Next we present the following lemma.

Lemma 4.9 Assume that $f \in S^{p} A P_{\omega}\left(\mathbb{R}^{+} \times X, X\right)$ and assume further that $f$ satisfies a Lipschitz condition in $X$ uniformly in $t \in \mathbb{R}^{+}$:

$$
\|f(t, x)-f(t, y)\| \leq L\|x-y\|
$$

for all $x, y \in X$ and $t \in \mathbb{R}^{+}$, where $L$ is a positive constant. Let $u \in A P_{\omega}\left(\mathbb{R}^{+}, X\right)$. Then the function $F: \mathbb{R}^{+} \rightarrow X$ defined by $F(t)=f(t, u(t))$ is asymptotically $\omega$-periodic in the Stepanov sense.

Proof Since $u \in A P_{\omega}\left(\mathbb{R}^{+}, X\right)$, we can write $u=v+l$, where $v \in P_{\omega}\left(\mathbb{R}^{+}, X\right)$ and $l \in C_{0}\left(\mathbb{R}^{+}, X\right)$. Moreover, $u \in S^{p} A P_{\omega}\left(\mathbb{R}^{+}, X\right), v \in S^{p} P_{\omega}\left(\mathbb{R}^{+}, X\right)$, and $l \in B S_{0}^{p}\left(\mathbb{R}^{+}, X\right)$. By Theorem 3.3 we obtain

$$
\left(\int_{t}^{t+1}\|u(s+n \omega)-v(s)\|^{p} d s\right)^{\frac{1}{p}} \rightarrow 0
$$

as $n \rightarrow \infty$ pointwise on $\mathbb{R}^{+}$.

Denote $K=\overline{\left\{v(t): t \in \mathbb{R}^{+}\right\}} ; K$ is a bounded set.

Since $f$ is asymptotically $\omega$-periodic in the Stepanov sense uniformly on bounded sets of $X$, there exists a function $g: \mathbb{R}^{+} \times X \rightarrow X$ with $g(t, x) \in S^{p}\left(\mathbb{R}^{+}, X\right)$ for each $x \in X$ such that

$$
\left(\int_{t}^{t+1}\|f(s+n \omega, x)-g(s, x)\|^{p} d s\right)^{\frac{1}{p}} \rightarrow 0
$$

as $n \rightarrow \infty$ pointwise on $\mathbb{R}^{+}$uniformly for $x \in K$.

By the Minkowski inequality, we have

$$
\begin{aligned}
& \left(\int_{t}^{t+1}\|f(s+n \omega, u(s+n \omega))-g(s, v(s))\|^{p} d s\right)^{\frac{1}{p}} \\
& \quad \leq\left(\int_{t}^{t+1}\|f(s+n \omega, u(s+n \omega))-f(s+n \omega, v(s))\|^{p} d s\right)^{\frac{1}{p}} \\
& \quad+\left(\int_{t}^{t+1}\|f(s+n \omega, v(s))-g(s, v(s))\|^{p} d s\right)^{\frac{1}{p}} \\
& \quad \leq L\left(\int_{t}^{t+1}\|u(s+n \omega)-v(s)\|^{p} d s\right)^{\frac{1}{p}}+\left(\int_{t}^{t+1}\|f(s+n \omega, v(s))-g(s, v(s))\|^{p} d s\right)^{\frac{1}{p}} .
\end{aligned}
$$

By (4.5) and (4.6), we get

$$
\left(\int_{t}^{t+1}\|f(s+n \omega, u(s+n \omega))-g(s, v(s))\|^{p} d s\right)^{\frac{1}{p}} \rightarrow 0
$$

as $n \rightarrow \infty$ pointwise on $\mathbb{R}^{+}$. By Theorem 3.3, one has $F \in S^{p} A P_{\omega}\left(\mathbb{R}^{+}, X\right)$. 
Now we can establish the main results of this paper.

Theorem 4.10 Assume that $A$ is sectorial of type $\mu<0$ with $0<\theta<\pi\left(1-\frac{\alpha}{2}\right)$. Let $f$ be asymptotically $\omega$-periodic in the Stepanov sense uniformly on bounded sets of $X$ and assume that $f$ satisfies a Lipschitz condition in $X$ uniformly in $t \in \mathbb{R}^{+}$:

$$
\|f(t, x)-f(t, y)\| \leq L\|x-y\|
$$

for all $x, y \in X$ and $t \in \mathbb{R}^{+}$, where $L$ is a positive constant. If $C M|\mu|^{-\frac{1}{\alpha}} \pi L<\alpha \sin \left(\frac{\pi}{\alpha}\right)$, where $C$ and $M$ are the constants in (4.2), then the problem (4.1) has a unique asymptotically $\omega$-periodic mild solution.

Proof Define the operator $\Gamma_{\alpha}$ on the space $A P_{\omega}\left(\mathbb{R}^{+}, X\right)$ by

$$
\left(\Gamma_{\alpha} u\right)(t)=S_{\alpha}(t) v_{0}+\int_{0}^{t} S_{\alpha}(t-s) f(s, u(s)) d s=S_{\alpha}(t) v_{0}+v_{\alpha}(t)
$$

By (4.2), we know that $S_{\alpha}(\cdot) v_{0} \in C_{0}\left(\mathbb{R}^{+}, X\right) \subset A P_{\omega}\left(\mathbb{R}^{+}, X\right)$. Let $u \in A P_{\omega}\left(\mathbb{R}^{+}, X\right)$. By Lemma 4.9 we get $f(s, u(s)) \in S^{p} A P_{\omega}\left(\mathbb{R}^{+}, X\right)$. Since $1<\alpha<2$, by (4.3) $\frac{C M}{1+|\mu| t^{\alpha}} \in L_{1}\left(\mathbb{R}^{+}\right)$and is nonincreasing. Combining with (4.2), Lemma 4.7 yields $v_{\alpha}(t) \in A P_{\omega}\left(\mathbb{R}^{+}, X\right)$. Therefore, $\Gamma_{\alpha}$ maps the space $A P_{\omega}\left(\mathbb{R}^{+}, X\right)$ into $A P_{\omega}\left(\mathbb{R}^{+}, X\right)$.

Finally, for $u, v \in A P_{\omega}\left(\mathbb{R}^{+}, X\right)$, one has

$$
\begin{aligned}
\left\|\left(\Gamma_{\alpha} u\right)(t)-\left(\Gamma_{\alpha} v\right)(t)\right\| & \leq \int_{0}^{t}\left\|S_{\alpha}(t-s)\right\|\|f(s, u(s))-f(s, v(s))\| d s \\
& \leq L \int_{0}^{t}\left\|S_{\alpha}(t-s)\right\|\|u(s)-v(s)\| d s \\
& \leq L\|u-v\|_{\infty} \int_{0}^{t}\left\|S_{\alpha}(s)\right\| d s \\
& \leq L\|u-v\|_{\infty} \int_{0}^{t} \frac{C M}{1+|\mu| t^{\alpha}} d s \\
& \leq \frac{C M|\mu|^{-\frac{1}{\alpha}} \pi L}{\alpha \sin \left(\frac{\pi}{\alpha}\right)}\|u-v\|_{\infty}
\end{aligned}
$$

which shows that

$$
\left\|\Gamma_{\alpha} u-\Gamma_{\alpha} v\right\|_{\infty} \leq \frac{C M|\mu|^{-\frac{1}{\alpha}} \pi L}{\alpha \sin \left(\frac{\pi}{\alpha}\right)}\|u-v\|_{\infty} .
$$

Hence $\Gamma_{\alpha}$ is a contraction mapping. To complete the proof of the theorem we only need to invoke the contraction mapping principle.

Theorem 4.11 Assume that $A$ is sectorial of type $\mu<0$ with $0<\theta<\pi\left(1-\frac{\alpha}{2}\right)$. Let $f$ be asymptotically $\omega$-periodic in the Stepanov sense uniformly on bounded sets of $X$ and assume that $f$ satisfies a Lipschitz condition:

$$
\|f(t, x)-f(t, y)\| \leq L_{f}(t)\|x-y\|
$$


for all $x, y \in X$ and $t \in \mathbb{R}^{+}$, where $L_{f}$ is a bounded measurable function. If $C M(1+$

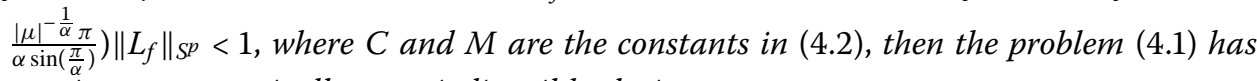
a unique asymptotically $\omega$-periodic mild solution.

Proof Proceeding as in the proof of Theorem 4.10, we define the operator $\Gamma_{\alpha}$ on the space $A P_{\omega}\left(\mathbb{R}^{+}, X\right)$ by (4.7). By Lemmas 4.9, 4.7 and a similar consideration in Theorem 4.10, $\Gamma_{\alpha}$ maps $A P_{\omega}\left(\mathbb{R}^{+}, X\right)$ into $A P_{\omega}\left(\mathbb{R}^{+}, X\right)$. Next we prove that $\Gamma_{\alpha}$ is a contraction. For $u, v \in$ $A P_{\omega}\left(\mathbb{R}^{+}, X\right)$, we have

$$
\begin{aligned}
\left\|\left(\Gamma_{\alpha} u\right)(t)-\left(\Gamma_{\alpha} v\right)(t)\right\| & \leq \int_{0}^{t}\left\|S_{\alpha}(t-s)\right\|\|f(s, u(s))-f(s, v(s))\| d s \\
& \leq \int_{0}^{t} \frac{C M}{1+|\mu|(t-s)^{\alpha}} L_{f}(s) d s\|u-v\|_{\infty} .
\end{aligned}
$$

Next we estimate the term $\int_{0}^{t} \frac{C M}{1+|\mu|(t-s)^{\alpha}} L_{f}(s) d s$. For $m \leq t<m+1, m \in \mathbb{N}$, we denote $l=$ $m+1-t$. Then one has

$$
\begin{aligned}
\int_{0}^{t} \frac{C M}{1+|\mu|(t-s)^{\alpha}} L_{f}(s) d s & =\int_{0}^{m+1-l} \frac{C M}{1+|\mu|(m+1-l-s)^{\alpha}} L_{f}(s) d s \\
& =\int_{l}^{m+1} \frac{C M}{1+|\mu|(m+1-s)^{\alpha}} L_{f}(s-l) d s .
\end{aligned}
$$

Define

$$
\widetilde{L_{f}}(s)= \begin{cases}0, & 0 \leq s<l \\ L_{f}(s-l), & s \geq l .\end{cases}
$$

We observe that $\left\|\widetilde{L_{f}}\right\|_{S^{p}}=\left\|L_{f}\right\|_{S^{p}}$. Then we have

$$
\begin{aligned}
\int_{0}^{t} \frac{C M}{1+|\mu|(t-s)^{\alpha}} L_{f}(s) d s & =\int_{0}^{m+1} \frac{C M}{1+|\mu|(m+1-s)^{\alpha}} \widetilde{L_{f}}(s) d s \\
& \leq \sum_{k=0}^{m} \int_{k}^{k+1} \frac{C M}{1+|\mu|(m+1-s)^{\alpha}} \widetilde{L_{f}}(s) d s \\
& \leq \sum_{k=0}^{m} \frac{C M}{1+|\mu|(m-k)^{\alpha}} \int_{k}^{k+1} \widetilde{L_{f}}(s) d s .
\end{aligned}
$$

By the Hölder inequality, we obtain $\int_{k}^{k+1} \widetilde{L_{f}}(s) d s \leq\left(\int_{k}^{k+1}{\widetilde{L_{f}}}^{p}(s) d s\right)^{\frac{1}{p}} \leq\left\|\widetilde{L_{f}}\right\|_{S^{p}}$. Thus,

$$
\begin{aligned}
\int_{0}^{t} \frac{C M}{1+|\mu|(t-s)^{\alpha}} L_{f}(s) d s & \leq \sum_{k=0}^{m} \frac{C M}{1+|\mu|(m-k)^{\alpha}}\left\|L_{f}\right\|_{S^{p}} \\
& \leq C M\left[1+\left(\int_{0}^{1}+\cdots+\int_{m-1}^{m}\right) \frac{1}{1+|\mu| t^{\alpha}} d t\right]\left\|L_{f}\right\|_{S^{p}} \\
& \leq C M\left(1+\int_{0}^{\infty} \frac{1}{1+|\mu| t^{\alpha}} d t\right)\left\|L_{f}\right\|_{S^{p}} \\
& =C M\left(1+\frac{|\mu|^{-\frac{1}{\alpha}} \pi}{\alpha \sin \left(\frac{\pi}{\alpha}\right)}\right)\left\|L_{f}\right\|_{S^{p}} .
\end{aligned}
$$


Therefore,

$$
\left\|\Gamma_{\alpha} u-\Gamma_{\alpha} v\right\|_{\infty} \leq C M\left(1+\frac{|\mu|^{-\frac{1}{\alpha}} \pi}{\alpha \sin \left(\frac{\pi}{\alpha}\right)}\right)\left\|L_{f}\right\|_{S^{p}}\|u-v\|_{\infty} .
$$

Hence $\Gamma_{\alpha}$ is a contraction mapping. To complete the proof of the theorem we only need to invoke the contraction mapping principle.

Finally, we provide an example to illustrate our results. Consider the following fractional differential equation.

\section{Example 4.12}

$$
\begin{cases}\partial_{t}^{\alpha} u(t, x)=\partial_{x}^{2} u(t, x)-\lambda u(t, x)+\partial_{t}^{\alpha-1} a(t) g(u(t, x)), & t \in \mathbb{R}^{+}, x \in[0, \pi] \\ u(t, 0)=u(t, \pi)=0, & t \in \mathbb{R}^{+} ; \\ u(0, x)=u_{0}(x), & x \in[0, \pi],\end{cases}
$$

where $1<\alpha<2, \lambda>0, u_{0} \in L^{2}[0, \pi]$, and $g: \mathbb{R}^{+} \rightarrow \mathbb{R}$ satisfies

(1) $|g(x)| \leq L_{1}|x|$;

(2) $|g(x)-g(y)| \leq L_{2}|x-y|$,

where $L_{1}$ and $L_{2}$ are positive constants. The function $a(t) \in S^{p} A P_{\omega}\left(\mathbb{R}^{+}, \mathbb{R}\right)$ satisfies $\sup _{t \in \mathbb{R}_{+}}|a(t)| \leq N$, where $N$ is a positive constant. Suppose $X=L^{2}[0, \pi]$ and let $A$ be the operator given by $A u=u^{\prime \prime}-\lambda u$ with domain $D(A)=\left\{u \in X: u^{\prime \prime} \in X, u(0)=u(\pi)=0\right\}$. It is well known that $A$ is a sectorial of type $\mu=-\lambda<0$. Let $f(t, \varphi)(s)=a(t) g(\varphi(s))$ for $\varphi \in X$ and $s \in[0, \pi]$. Then (4.8) can be transformed into (4.1), where $u(t)(x)=u(t, x)$ for $t \in \mathbb{R}^{+}$, $x \in[0, \pi]$. Since $a \in S^{p} A P_{\omega}\left(\mathbb{R}^{+}, \mathbb{R}\right)$, by Theorem 3.3 there exists a function $b \in S^{p} P_{\omega}\left(\mathbb{R}^{+}, \mathbb{R}\right)$ such that

$$
\left(\int_{t}^{t+1}|a(s+n \omega)-b(s)|^{p} d s\right)^{\frac{1}{p}} \rightarrow 0
$$

as $n \rightarrow \infty$ pointwise on $\mathbb{R}^{+}$. Let $g(t, \varphi)(s)=b(t) g(\varphi(s))$. For each $t \in \mathbb{R}^{+}$, we have

$$
\begin{aligned}
\|f(t+n \omega, \varphi)-g(t, \varphi)\| & =\left(\int_{0}^{\pi}|a(t+n \omega) g(\varphi(s))-b(t) g(\varphi(s))|^{2} d s\right)^{\frac{1}{2}} \\
& =|a(t+n \omega)-b(t)|\left(\int_{0}^{\pi}|g(\varphi(s))|^{2} d s\right)^{\frac{1}{2}} \\
& \leq L_{1}|a(t+n \omega)-b(t)|\left(\int_{0}^{\pi}|\varphi(s)|^{2} d s\right)^{\frac{1}{2}} \\
& \leq L_{1}|a(t+n \omega)-b(t)|\|\varphi\| .
\end{aligned}
$$

Then for each $t^{\prime} \in \mathbb{R}^{+}$, one has

$$
\begin{aligned}
& \left(\int_{t^{\prime}}^{t^{\prime}+1}\|f(t+n \omega, \varphi)-g(t, \varphi)\|^{p} d t\right)^{\frac{1}{p}} \\
& \quad \leq L_{1}\|\varphi\|\left(\int_{t^{\prime}}^{t^{\prime}+1}|a(t+n \omega)-b(t)|^{p} d t\right)^{\frac{1}{p}} .
\end{aligned}
$$


Denote $\Omega_{M}=\{\varphi \in X:\|\varphi\| \leq M\}$. Then we have

$$
\left(\int_{t^{\prime}}^{t^{\prime}+1}\|f(t+n \omega, \varphi)-g(t, \varphi)\|^{p} d t\right)^{\frac{1}{p}} \rightarrow 0
$$

as $n \rightarrow \infty$ pointwise on $\mathbb{R}^{+}$uniformly for $\varphi \in \Omega_{M}$. Clearly, for each $\varphi \in X, g(t, \varphi) \in$ $S^{p} P_{\omega}\left(\mathbb{R}^{+}, X\right)$. So $f \in S^{p} A P_{\omega}\left(\mathbb{R}^{+} \times X, X\right)$.

On the other hand, we have

$$
\begin{aligned}
\|f(t, u)-f(t, v)\| & =\left(\int_{0}^{\pi}|a(t) g(u(s))-a(t) g(v(s))|^{2} d s\right)^{\frac{1}{2}} \\
& \leq N L_{2}\left(\int_{0}^{\pi}|u(s)-v(s)|^{2} d s\right)^{\frac{1}{2}} \\
& \leq N L_{2}\|u-v\| .
\end{aligned}
$$

So when $L_{2}$ is small enough, by Theorem 4.10 the problem (4.8) has a unique asymptotically $\omega$-periodic mild solution.

Remark 4.13 This example is motivated by the example in [13]. For a specific result, we can define $g(x)$ by $\sin (x)$ and choose $a(t)$ as Example 3.2.

\section{Competing interests}

The authors declare that they have no competing interests.

\section{Authors' contributions}

All authors contributed equally to the manuscript. All authors read and approved the final manuscript.

\section{Acknowledgements}

This research was supported by the National Natural Science Foundation of China (No. 11071048). The authors are thankful to the referees for their careful reading of the manuscript and insightful comments.

Received: 30 October 2014 Accepted: 4 February 2015 Published online: 01 March 2015

\section{References}

1. Agarwal, RP, Cuevas, C, Soto, H, El-Gebeily, M: Asymptotic periodicity for some evolution equations in Banach spaces. Nonlinear Anal. 74, 1769-1798 (2011)

2. Diagana, T: Almost Automorphic Type and Almost Periodic Type Functions in Abstract Spaces. Springer, New York (2013)

3. Fan, Z, Liang, J, Xiao, TJ: On Stepanov-like (pseudo) almost automorphic functions. Nonlinear Anal. 74, 2853-2861 (2011)

4. Berger, A, Siegmund, S, Yi, Y: On almost automorphic dynamics in symbolic lattices. Ergod. Theory Dyn. Syst. 24, 677-696 (2004)

5. Nicola, SHJ, Pierri, M: A note on S-asymptotically periodic functions. Nonlinear Anal., Real World Appl. 10, 2937-2938 (2009)

6. Henríquez, HR, Pierri, M, Táboas, P: On S-asymptotically $\omega$-periodic functions on Banach spaces and applications. J. Math. Anal. Appl. 343, 1119-1130 (2008)

7. Henríquez, HR, Pierri, M, Táboas, P: Existence of S-asymptotically $\omega$-periodic solutions for abstract neutral equations. Bull. Aust. Math. Soc. 78, 365-382 (2008)

8. de Andrade, B, Cuevas, C: S-Asymptotically $\omega$-periodic and asymptotically $\omega$-periodic solutions to semi-linear Cauchy problems with non-dense domain. Nonlinear Anal. 72, 3190-3208 (2010)

9. Pierri, M: On S-asymptotically $\omega$-periodic functions and applications. Nonlinear Anal. 75, 651-661 (2012)

10. Wang, RN, Xiang, QM, Zhou, Y: Asymptotically periodic solutions to nonlocal Cauchy problems governed by compact evolution families. J. Math. Sci. 197(1), 14-28 (2014)

11. Henríquez, HR, Cuevas, C, Caicedo, A: Asymptotically periodic solutions of neutral partial differential equations with infinite delay. Commun. Pure Appl. Anal. 12(5), 2031-2068 (2013)

12. Pierri, M, Rolnik, V: On pseudo S-asymptotically periodic functions. Bull. Aust. Math. Soc. 87, 238-254 (2013)

13. Cuevas, C, de Souza, JC: S-Asymptotically $\omega$-periodic solutions of semilinear fractional integro-differential equations. Appl. Math. Lett. 22, 865-870 (2009) 
14. Cuevas, C, de Souza, JC: Existence of S-asymptotically $\omega$-periodic solutions for fractional order functional integro-differential equations with infinite delay. Nonlinear Anal. 72, 1683-1689 (2010)

15. Cuevas, C, Pierri, M, Sepulveda, A: Weighted S-asymptotically $\omega$-periodic solutions of a class of fractional differential equations. Adv. Differ. Equ. 2011, Article ID 584874 (2011)

16. Cuevas, C, Henríquez, HR, Soto, H: Asymptotically periodic solutions of fractional differential equations. Appl. Math. Comput. 236, 524-545 (2014)

17. Ponce, R: Bounded mild solutions to fractional integro-differential equations in Banach spaces. Semigroup Forum 87, 377-392 (2013)

18. Xia, Z: Asymptotically periodic solutions of semilinear fractional integro-differential equations. Adv. Differ. Equ. 2014, Article ID 9 (2014)

19. Lizama, C, N'Guérékata, GM: Bounded mild solutions for semilinear integro differential equations in Banach spaces. Integral Equ. Oper. Theory 68, 207-227 (2010)

20. Agarwal, RP, de Andrade, B, Cuevas, C: On type of periodicity and ergodicity to a class of fractional order differential equations. Adv. Differ. Equ. 2010, Article ID 179750 (2010)

21. Caicedo, A, Cuevas, C, Mophou, GM, N'Guérékata, GM: Asymptotic behavior of solutions of some semilinear functional differential and integro-differential equations with infinite delay in Banach spaces. J. Franklin Inst. 349, $1-24$ (2012)

22. Henríquez, HR: Asymptotically periodic solutions of abstract differential equations. Nonlinear Anal. 80, 135-149 (2013)

23. Cuesta, E: Asymptotic behaviour of the solutions of fractional integro-differential equations and some time discretizations. Discrete Contin. Dyn. Syst., Ser. B 2007, 277-285 (2007)

24. Bazhlekova, E: Fractional evolution equation in Banach spaces. Ph.D. thesis, Eindhoven University of Technology (2001)

25. Mophou, GM, N'Guérékata, GM: Existence of the mild solution for some fractional differential equations with nonlocal conditions. Semigroup Forum 79, 315-322 (2009)

26. Cuevas, C, Lizama, C: Almost automorphic solutions to a class of semilinear fractional differential equations. Appl. Math. Lett. 21, 1315-1319 (2008)

27. Anh, W, Mcvinish, R: Fractional differential equations driven by Lévy noise. J. Appl. Math. Stoch. Anal. 16(2), 97-119 (2003)

28. Lunardi, A: Analytic Semigroups and Optimal Regularity in Parabolic Problems. Progress in Nonlinear Differential Equations and Their Applications, vol. 16. Birkhäuser, Basel (1995)

29. Keyantuo, V, Lizama, C: On a connection between powers of operators and fractional Cauchy problems. J. Evol. Equ. $12,245-265$ (2012)

30. Podlubny, I: Fractional Differential Equations. Academic Press, New York (1999)

31. Carpinteri, A, Cornetti, P: A fractional calculus approach to the description of stress and strain localization in fractal media. Chaos Solitons Fractals 13, 85-94 (2002)

\section{Submit your manuscript to a SpringerOpen ${ }^{\circ}$ journal and benefit from:}

- Convenient online submission

Rigorous peer review

- Immediate publication on acceptance

- Open access: articles freely available online

- High visibility within the field

- Retaining the copyright to your article 